\title{
Writing for Clinical Orthopaedics and Related Research
}

\author{
Richard A. Brand MD
}

(C) The Association of Bone and Joint Surgeons 2008

Because of the growing need for publication space, Clinical Orthopaedics and Related Research (CORR) was established in 1953 by the Association of Bone and Joint Surgeons [13] to provide an alternative source of publication to the Journal of Bone and Joint Surgery (then the only American orthopaedic journal [8]). CORR always has striven to provide readers with high-quality peer-reviewed articles in the form of original research and survey material. High quality depends on characteristics of the work and on the reporting. While there is no lack of excellent material on medical reporting and writing $[7,11,12,15$, $17,31]$, this article (and its predecessor on which this is based [6]) is directed to CORR contributors. Toward that end, I shall provide updated guidelines to our authors for an approach to effective reporting.

Standards of reporting, no less than standards of scientific conduct (ethics) and standards of acceptable scientific methods, change. Although ethics has always played a critical part in science and scientific reporting, recent societal and regulatory expectations impose certain new requirements, while scientific advances require others. These changes have stimulated considerable discussion $[1,2,9,14$,

The author certifies that he has no commercial associations (eg, consultancies, stock ownership, equity interest, patent/licensing arrangements, etc) that might pose a conflict of interest in connection with the submitted article. (Adapted with permission of Lippincott Williams and Wilkins from Brand RA. Writing for Clinical Orthopaedics and Related Research. Clin Orthop Relat Res. 2003;413:1-7.)

\section{R. A. Brand $(\square)$}

Clinical Orthopaedics and Related Research, 1600 Spruce Street, Philadelphia, PA 19103, USA

e-mail: dick.brand@clinorthop.org
22-26] and CORR contributors are specifically directed to the publications of the International Committee of Medical Journal Editors [16] and The Committee on Publication Ethics for general guidelines [1,2]. CORR adheres to these evolving guidelines, particularly regarding ethical issues. Scientific advances in recent years include use of contemporary outcome measures, more sophisticated statistical approaches, and increasing use and reporting of well-formulated research plans (particularly in clinical research such as the CONSORT guidelines [18]). Although I shall not detail these changing standards of reporting in my review, I shall explicitly note several issues.

Scientific writing, no less than any other form of writing, reflects a demanding creative process, not merely an act: the process of writing changes thought. The quality of a report, however, depends on the quality of thought in the study design and the rigor of conduct of the research. Well-posed questions or hypotheses intimately and inexorably interrelate with study design and analysis. Well-posed hypotheses or questions imply a study design and a study design implies hypotheses or questions.

The effectiveness of a report relates to focus and brevity. Attention to a few points will allow authors to focus on critical issues. Brevity is achieved in part by avoiding repetition (with a few exceptions to be noted), clear style [17], and proper grammar [27, 31]. Few original scientific articles need be longer than 3000 words. Longer articles (eg, 4000-5000 words) may be warranted if substantially novel methods are reported, or if the article reflects a systematic survey of literature. Although writers should avoid redundancy, effectively communicating critical information often means limited repetition of the questions (or hypotheses or key issues) and answers. The questions/hypotheses should appear in the Abstract, Introduction, and Discussion, and the answers 
should appear in the Abstract, Results, and Discussion. Apart from these exceptions, authors should not repeat material.

Styles of writing are as numerous as authors, although most journals publish guidelines for formatting a manuscript, and many have more or less established writing styles (eg, the American Medical Association Manual of Style) [3]. CORR uses the AMA style as a general guideline. However, few scientific and medical authors have the time to learn these styles. Therefore, within the limits of proper grammar and clear, effective communication, and our guidelines, we allow individual styles.

I shall outline the various elements of a traditional archival report (Introduction, Materials and Methods, Results, Discussion) and suggest a logical flow for each. Each of these sections should contain unique information, and the sort of information required for one should not appear in the other; often authors mix rationale and methods, methods and results, or results and discussion. Much of this information relates to clinical studies, but the principles apply to basic reports as well. Some reports, such as surveys and systematic literature reviews or meta-analyses, require individualized structures, although I shall also describe some generalizations applicable to these studies.

\section{Introduction (500 words)}

The Introduction, although typically the shortest of sections, critically states the issues and formulates the rationale for the questions or hypotheses. Its organization might differ somewhat for a clinical report, a study of new scientific data, or a description of a new method. Most studies, however, are published to (1) report entirely novel findings (only occasionally with substantive basic or clinical studies and rarely a case report), (2) confirm previously reported work (eg, case reports, small preliminary series) when such confirmation remains questionable, or (3) introduce or address controversies in the literature when data and/or conclusions conflict. Apart from certain surveys and other special articles, one of these three purposes generally should be apparent (and often explicit) in the Introduction. The first paragraph should introduce the general topic or problem and suggest its importance, a second and perhaps a third paragraph should provide the rationale for each question or hypothesis, and a final paragraph should state the questions, hypotheses, or purposes.

One may think of formulating rationale and hypotheses as Aristotelian logic (a modal syllogism) taking the form: If A, $\mathrm{B}$, and $\mathrm{C}$, then $\mathrm{D}, \mathrm{E}$, or $\mathrm{F}$. The premises $\mathrm{A}, \mathrm{B}$, and $\mathrm{C}$ reflect accepted facts (rationale) whereas $\mathrm{D}, \mathrm{E}$, or $\mathrm{F}$ reflect logical outcomes or predictions (questions or hypotheses). The premises best come from published data, but when data are not available, published observations (typically qualitative), logical argument, or consensus of opinion can be used. The strength of these premises is roughly in descending order from data to observations or argument to opinion. D, E, or F reflect logical consequences. For any set of observations, any number of explanations (D, E, or F) logically follow. Therefore, when formulating hypotheses (explanations), researchers designing experiments and reporting results should not be wed to a single explanation.

With the rare exception of truly novel material, when establishing rationale authors should provide representative (although not necessarily exhaustive) literature. Such rationale places a work within the body of literature. Writers should merely state their premises and provide relevant citations and avoid mention of authors' names or description of cited works; this places emphasis on ideas rather than on investigators and studies. The exceptions to this approach include a description of past methods when essential to developing rationale for a new method or a mention of authors' names when important to establish historical precedent. Amplification of the citations may follow in the Discussion when appropriate.

New treatment approaches require a specific sort of rationale: new interventions of any sort are intended to solve certain problems with previous approaches and those problems should be explicitly noted. For example, new implants (unless conceptually novel) typically will be designed according to certain criteria to eliminate problems with previous implants. Therefore, if the intent is to report a new treatment and/or its outcome, the premises of the study should include those explicitly stated problems (with ranges of incidence from the literature when possible) and they should be properly referenced.

The final paragraph logically flows from the earlier ones and should explicitly state the questions or hypotheses to be addressed in terms of the study variables (independent, dependent). Any issue not posed in terms of study variables cannot be meaningfully addressed. Assignment of a Level of Evidence in clinical studies requires a primary research question, which in turn requires a key outcome variable. Focus demands authors avoid answers to unposed questions and answers that are well described in the literature (eg, reporting of heterotopic ossification rates when the question is whether an implant minimizes stress shielding). Descriptive purposes (eg, "The purpose of our study was to report the results...") are appropriate only for Level IV studies (one cohort in therapeutic studies) and only when the information is novel, an uncommon situation since typically some information has been previously reported, and the data will merely confirm or refute previously published data or will address or introduce a controversy. I reiterate any well-posed question or hypothesis will be reflected by the study design and statistical analysis; every statistical analysis necessarily implies specific questions. 


\section{Materials and Methods (1000-1500 words)}

In principle, the Materials and Methods should contain adequate detail for another investigator to replicate the study. This principle applies to surveys or systematic reviews as well as to archival manuscripts since search strategies should be repeatable. In practice, such detail is often neither practical nor desirable because many methods will have been published previously (and in greater detail) and because long descriptions violate the principle of brevity. Nonetheless, the Materials and Methods section typically will be the longest section.

The Materials and Methods should flow in the approximate chronological order in which the study was conducted. For a clinical study, these elements entail (1) a study design (including key variables), (2) a power analysis (for all studies statistically comparing two or more groups), (2) key patient demographics (ie, those which could be confounding variables), (3) a description of surgery if any, (4) postoperative care, (5) a description of valid and reliable methods to measure study independent variables (eg, followup clinical, radiographic, or histologic outcomes in a clinical study and independent variables in all statistical analyses), and (6) their statistical analysis. Basic studies obviously do not contain patient demographics or surgery or postoperative care but generally require the other elements.

At the outset, the reader should grasp the basic study design. The study design should complement the questions or hypotheses just raised and must similarly state the key study variables. Level IV therapeutic studies contain a single cohort, but Level I to III studies necessarily describe two or more cohorts (independent variables); these must be outlined in the study design, as well as the key dependent variables. Level I to III studies also require a power analysis of the key outcome variable of the primary research question. This analysis should be based upon a clinically meaningful (and not merely statistically significant) effect size. That is, what difference in that variable or measure would result in a substantially different clinical outcome? A judgment as to meaningful differences should be based upon the literature when available or an educated guess when not; readers may judge whether they agree or disagree with the choice.

The first paragraph or two should also contain, in addition to the study design (and power analysis when appropriate), all relevant clinical data (Appendix 1). For a given study, not all information may be essential, but authors can use the types of data in the appendix as a checklist. Minimum followup should be sufficient to meaningfully address the questions being posed. This will necessarily differ depending upon the question. Questions relating to postoperative mortality rates may require, for example, only 30 to 90 days of followup, while those for questions relating to implant loosening may require 5 years. Authors must state the minimum followup time followed by the mean (or median when more appropriate) and range.

Clinical reports must state inclusion and exclusion criteria and whether the series is consecutive or selected; if selected, criteria for selection should be stated. The reader should understand from this description all potential sources of bias such as referral, diagnosis, exclusion, recall, or treatment bias. This includes the manner in which investigators selected the patients. Consecutive inclusion implies all patients with a given diagnosis (not typically a given treatment) are included, while selective implies patients with a given diagnosis but selected according to certain explicit criteria (eg, state of disease, choice of treatment). Often in a surgical series, patients to be reported are selected for a given treatment (eg, type of surgery or implant) rather than a given diagnosis. This occurs when some patients with the diagnosis have the treatment in question, but other patients treated during the same time frame with the same diagnosis have a different treatment or treatments; in this case, authors must specify the criteria used to select and exclude the treatment in question. Referral bias will typically be known to readers based upon the institution or institutions from which the study arises, although occasionally referral bias requires amplification in Materials and Methods.

All patient studies must include a description of potentially confounding demographic information at the time of entry into the study and at the time of surgery; this information should appear in the first paragraph or two. With a single cohort, this information will reflect referral or selection bias. With more than one cohort (ie, Level I-III studies), authors should provide a statistical analysis of these potentially confounding baseline variables between the groups; thus the reader immediately knows whether the groups are biased in any important way. Potentially biasing differences should be described not only statistically but also in clinically meaningful terms.

Missing data confound many statistical analyses. Authors, particularly those of retrospective reviews, should note what data are missing and in the statistical descriptions should note how they dealt with missing data.

Any treatment (including surgery) should be briefly described, particularly when surgeons apply unique approaches or when all patients did not undergo essentially identical procedures. Previously described approaches require only brief mention with citations to those methods.

All relevant aspects of posttreatment followup care should be described, whether nonsurgical or surgical. If that treatment might reasonably be expected to influence outcome, it should be described in some detail. Authors must note whether the treatment was uniform among all patients or varied. If varied, they should specify the 
indications for treating patients in varying ways and, if the study involves multiple cohorts, whether the treatment applications statistically differed between the cohorts.

If authors use statistical analysis, a paragraph should appear at the end of Materials and Methods stating all statistical tests used. Statistical tests imply specific questions or hypotheses, so the methods and their descriptions should be coherent with and in the same order as the questions or hypotheses posed in the Introduction. Authors must specify the variables analyzed with each test. All statistical tests are associated with assumptions, and when it is not obvious the data would meet those assumptions, the authors either should provide the supporting data (eg, data are normally distributed, variances in groups are similar) or use alternative tests. Although it is common to choose a level of alpha of 0.05 and a beta of 0.80 , these levels are somewhat arbitrary and not always appropriate. In the case where the implications of an error are very serious (eg, missing the diagnosis of a cancer), different alpha and beta levels might be appropriate in the study design to assess clinical or biological significance. Sterne and Davey Smith [29] provide historical and theoretical reasons why attempting to suggest something is important or not based on an arbitrary threshold level was not the intent of the developers and is inappropriate. We suggest readers avoid such thresholds and rather state the exact probability value in Results to demonstrate the strength of the evidence.

\section{Results (500 words)}

If the questions or issues have been adequately focused in the Introduction, the Results section need not be long. Authors may need an initial paragraph or two to persuade the reader of the validity of the methods and should have one paragraph addressing each explicitly raised question or hypothesis; finally, an additional paragraph or two might be useful to report new and unexpected findings. That is, authors should provide a one-to-one correspondence of questions and answers. Patient information in Results should be limited to that at the time of followup to answer the questions raised in the final paragraph of the Introduction while patient information at the time of entry into the study or at the time of surgery or before assessing the independent variables should appear in Materials and Methods (these descriptions, while often in the form of data, should be considered materials).

The first (topic) sentence of each paragraph should state the point or answer the question. Well-posed questions can be unambiguously answered "yes" or "no," and wellformulated hypotheses can be unambiguously confirmed or refuted. When the reader considers only the first sentence in each paragraph in Results, the logic of the authors' interpretations should be clear. Parenthetic reference to all figures and tables forces the writer to textually state the interpretation of the data; the important material is the authors' interpretation of the data, not the data.

Statistical reporting of data deserves special consideration. Stating some outcome is increased or decreased (or greater or lesser) and parenthetically stating the $\mathrm{p}$ (or other statistical) value immediately after the comparative terms more effectively conveys information than merely stating two values and then providing a probability value (in the same or a following sentence) without stating how or in which direction the two values differ. Avoiding the terms "statistically different" or "significantly different" but providing the exact probability value lets the reader determine whether they will consider the statistical value biologically or clinically important, regardless of statistical significance. Although a matter of philosophy and style, actual $\mathrm{p}$ values convey more information than stating a value less than some preset level. Furthermore, as Motulsky notes, "When you read that a result is not significant, don't stop thinking . . . First, look at the confidence interval . . . Second, ask about the power of the study to find a significant difference if it were there" [20]. This approach will give the reader a much greater sense of biological or clinical importance. Authors should avoid making inferences from nonsignificant trends unless they believe their study underpowered to answer that question; in that case, they should provide a power analysis.

\section{Discussion (1000 words)}

The Discussion in a CORR article should contain specific elements: a restatement of the problem or question, an exploration of limitations and assumptions, a comparison and/or contrast with information (data, opinion) in the literature, and a synthesis of the comparison and the author's new data to arrive at conclusions. The restatement of the problem or questions need be only brief for emphasis.

I prefer an exploration of assumptions and limitations immediately follow the brief introductory paragraph in Discussion rather than appear at the end because interpreting what will follow depends on these limitations. Failure to explore limitations suggests the author(s) either do not know or choose to ignore them, potentially misleading the reader. Exploration of these limitations need be only brief, but all critical issues must be raised, and the reader should be persuaded by logical argument they do not jeopardize the conclusions. Given the expense and effort for substantial prospective studies, it is not surprising most published clinical studies are retrospective. Such studies often are criticized unfairly for being retrospective, but that does not necessarily negate either validity or value of a 
study. Carefully designed retrospective studies have always provided and will continue to provide most of the information on which clinicians make decisions. However, authors reporting retrospective studies should describe any specific limitations relating to their study; these might include loss to followup, difficulty matching cohorts (if more than one), missing data, and the various forms of bias more common with retrospective studies.

Next, the authors should compare and/or contrast their data with data reported in the literature. Some of these reports may include those cited as rationale in the Introduction. Quantitative comparisons most effectively persuade the reader the data in the study are in the ballpark, and tables or figures efficiently convey that information. However, because of the peculiarities of each study, the data or observations might not be strictly comparable, but even in such cases it would be unusual if the literature would not contain at least trends or opinions for comparison. Discrepancies should be stated and explained when possible; when an explanation of a discrepancy is not clear, that also should be stated. Conclusions based solely on data in the paper seldom are warranted because the literature almost always contains previous information. The quality of any report will depend on the substantive nature of these comparisons.

Finally, the author(s) should synthesize their data with that in the literature. No critical data should be overlooked because contrary data might effectively refute an argument. (From a logical point of view, many consistent observations do not confirm an explanation because a single inconsistent observation can disprove an explanation.) That is, the final conclusions must be consistent not only with the new data presented but also with that in the literature.

\section{Surveys, Systematic Reviews, Meta-analyses}

The format for these three types of reports necessarily differs from those reporting original data. However, many of the principles noted above apply. These articles still require an Abstract, an Introduction, and a Discussion. The Introduction still requires focused issues and a rationale for those issues. Authors should convey to readers the unique aspects of their surveys that distinguish them from other available material (eg, monographs, book chapters). The issues should be posed in the final paragraph of the Introduction. As with an archival article reporting original material, the Introduction to a survey typically need not be longer than four paragraphs. Longer Introductions tend to lose focus, so the reader is not sure what novel information will be presented.

The sections after the Introduction and before the Discussion will be unique to the particular survey but need to be organized in a coherent fashion. Headings (and subheadings when appropriate) should follow parallel (grammatical) construction and reflect explicitly raised questions (Introduction) and generally reflect analogous topics (eg, diagnostic categories, choices of methods, choices of surgical interventions). If the reader considered only the headings, the logic of the survey (as reflected in the Introduction) should be clear.

Systematic reviews and meta-analyses are special sorts of studies meeting specific criteria of conduct $[5,19,30,32]$. In these articles the questions must be carefully defined and literature searches are conducted with inclusion criteria and stated search criteria including field tags appropriate to the questions. A Materials and Methods section will specify all databases searched, including hand searches of articles later identified as appropriate for initial consideration. The number of articles found by each search must be specified as should be the number excluded by explicit exclusion criteria. Authors must then state how they selected the final group of articles reviewed. These procedures allow an independent individual to more or less replicate the review (realizing many if not most sources today are electronic and the number of articles for a given search criterion is in continual flux). For clinical articles, authors must state whether and how they judged study quality; tables are often helpful in summarizing study quality. Meta-analyses require, in addition to those elements for a systematic review, additional requirements for analysis [5, 19]. These include a requirement for judging and accounting for study quality. Systematic reviews and meta-analyses should contain a traditional Results section that focuses on answers to the questions addressed in the Introduction (and not the material used to arrive at the answers). That is, a systematic review should synthesize the material and not merely provide summaries of individual articles. Tables or appendices may be used to provide summaries of material when necessary to support and supplement the synthesis. If select individual articles require summaries, they should appear in Materials and Methods, not in Results.

The Discussion sections of surveys, systematic reviews, and meta-analyses synthesize the reviewed literature into a coherent whole and within the context of the novel issues stated in the Introduction. The limitations should reflect those of the literature in general, however, in addition to those of a given study. Those limitations will relate to gaps in the literature, which preclude more or less definitive assessment of diagnosis or selection of treatment, for example. Controversies in the literature should be briefly explored. Only by exploring limitations will the reader appropriately place the literature in perspective. Authors should end the Discussion by summary statements similar to those that will appear at the end of the Abstract in abbreviated form.

In general, a survey requires a more extensive literature review than an archival article, although this depends on 
the topic and breadth of available literature. Some topics (eg, osteoporosis) could not be comprehensively referenced, even in an entire monograph. However, authors need to ensure a survey is representative of the entire body of literature, and when that body is large, many references are required.

\section{Acknowledgments}

Many individuals contribute to a study. Those who make important contributions should be so acknowledged. In conjunction with internationally recognized standards for authorship, CORR generally limits authorship to five individuals since more than that rarely make substantive contributions to at least three major elements of a study and its report. We make occasional exceptions for multidisciplinary studies or higher level (of evidence) multiinstitutional studies. Individuals making important contributions but not fulfilling criteria for authorship should be acknowledged.

\section{Abstract (200 words)}

Generally, the Abstract should be written after the entire manuscript is completed. The reason relates to how the process of writing changes thought and perhaps even intent. Only after careful consideration of the data and a synthesis with the literature can author(s) write an effective abstract.

Many readers, professional and lay alike, now access medical and scientific information via Web-based databases rather than browsing hard copy material. Regardless of access, since the reader's introduction occurs through titles and abstracts, substantive titles and abstracts more effectively capture a reader's attention. Because of lay access to abstracts, these should be written in accessible language and draw conclusions that would not be misinterpreted by a lay reader. Whether a reader will examine an entire article often will depend on an abstract with compelling information. A compelling abstract contains the questions or purposes, the methods, the results (most often quantitative data), and the answers to the questions (conclusions). Each of these may be conveyed in one or two statements. Comments such as "this report describes..." convey little useful information.

Clinical studies (ie, those involving data from patients) require a Level of Evidence when they relate to treatment, diagnosis, prognosis, or economic decisions. Authors must provide below the Abstract a Level of Evidence and study description for the primary research question (our Web site contains the guidelines).

\section{Title Page}

While the Abstract is important in capturing a reader's attention, the title is likely even more important owing to Internet methods of searching and browsing. Declarative titles raising or answering questions in a few brief words will far more likely do this than titles merely pointing to the topic. A title such as "Bisphosphonates Reduce Bone Loss" effectively conveys the main message and readers will more likely remember that message and read (and cite) the paper than they might if the title were "The Effect of Bisphosphonates on Bone Loss." As noted earlier, studies with multiple cohorts or groups statistically analyzed for differences require a power analysis of effect size based on meaningful biological or clinical differences; authors should consider a title based upon those key questions or hypotheses used for the power analysis. CORR generally limits titles to 80 characters including spaces.

CORR requires authors to acknowledge potential conflicts of interest. Our required cover letter contains four choices, one of which authors must select. This statement then becomes the basis for a standard statement that in our decision letters we request authors place on their Title Page.

When reporting studies involving human or animal subjects, authors must obtain prior approval of the institutional review board or ethics committees according to the laws and regulations of their countries. Informed consent for participation in a clinical study (a different form of consent from that required for treatment) must be stated where appropriate. In the United States, Institutional Review Board approval is required for studies using any information with patient identifiers, even if patients are not seen, although expedited review may be appropriate [21]. Similarly, animal studies require approval of institutional animal welfare committees. Such approval must be stated on the Title Page and may be stated in the first paragraph of Materials and Methods.

Finally, the Title Page must contain full contact information of the corresponding author.

\section{References}

References should derive primarily from peer-reviewed journals, standard textbooks or monographs, or wellaccepted and stable electronic sources (eg, NIH or FDA Web sites). For citations dependent on interpretation of data, authors generally should use only high-quality peerreviewed sources. Abstracts and submitted articles should not be used because many in both categories frequently do not pass peer review [4, 10, 28]. Accepted articles in press in peer-reviewed journals may be used if the anticipated date of publication is within a time frame for the final 
citation to be completed in CORR page proofs; if in press articles contain methods or data crucial to interpret material in a submitted manuscript, authors should include a copy in the submission for review by referees and editors.

CORR uses a modified AMA reference style. The modifications include listing of all authors, rather than only the first six authors, italicization of journal titles, and elimination of issue number.

\section{Figures and Tables}

Figures and tables should generally complement, not duplicate, material in the text. They compactly present information that would be difficult to describe in text form. (Material, which may be succinctly described in text, should rarely be placed in tables or figures.) Clinical studies, for example, often contain complementary tables of demographic data, which, although important for interpreting the results, are not critical for the questions raised in the paper. Well-focused papers contain only one or two tables or figures for every question or hypothesis explicitly posed in the Introduction. Additional material may be used for unexpected results.

Well-constructed tables are self-explanatory and require only a brief title. Every column must contain a header (with units when appropriate). Brief footnotes may be necessary to explain abbreviations or levels of statistical significance.

Figures typically need some explanation, including the meaning of symbols. Some figures will illustrate methods and may not require more than a brief explanation. Data figures should be provided only to address explicitly raised questions or hypotheses (Introduction) or unanticipated findings; data not directly addressing such questions should be avoided. In addition to whatever data descriptions are required, a figure legend should contain the major point within the framework of the questions raised. A reader should be able to read the questions in the last paragraph of the Introduction and then find the answers in the first sentence of each paragraph in Results and in the figure legends. Illustrations for a single patient should all have the same number and be labeled "A," "B," "C," etc. Each figure requires a separate legend and presumably makes a separate point. Legends should be written in complete sentences.

\section{Summary}

Effective writing demands three elements: focus, logical flow, and brevity. Focus is achieved by posing clear questions or hypotheses in terms of study variables and then describing the measures required only for those variables and reporting data only related to the questions or hypotheses. In the course of investigations, authors frequently collect far more data than is required for specific questions and they find it painful to discard data acquired with often great effort and expenditure of resources. Yet, attempting to report more data than required to address the questions or hypotheses merely loses readers. Logical flow is attained by following a clear structure. The four elements of an archival paper provide overall structure, but each element must also be logically structured: the Introduction with rationale and questions posed in order of importance, the Materials and Methods with a more or less chronological order of obtaining the materials and then describing the methods of assessing each variable, the Results by answering the questions in order of importance and appearance, and finally a Discussion that synthesizes the key data for each question with that in the literature. Authors should ensure each section contains only material appropriate for that section; for example, methods should not be described in Results. Brevity is achieved by avoiding repetition of material, eliminating data and discussion not required for the questions, and ensuring each section contains only the crucial information. Through these principles, authors can achieve a wider and more interested audience.

\section{Practical Tips}

1. Focus the data on addressing explicit hypotheses or questions; avoid the urge to report data not directly related to the hypotheses or questions, regardless of how painstaking it was to collect.

2. Read only the first sentence in each paragraph throughout the text to ascertain whether those statements contain all critical material and the logical flow is clear.

3. Avoid in the Abstract comments such as "this report describes..." Such statements convey no substantive information for the reader.

4. Avoid references and statistical values in the Abstract.

5. Avoid using the names of cited authors except to establish historical precedent. Instead, state the point documented in the article or articles and provide citation. For the most part, it is the data or the conclusions of the authors the reader finds crucial in scientific arguments, not the names of authors.

6. Avoid in the final paragraph of the Introduction descriptive purposes such as "we report our data..." Such statements fail to focus the reader's (and writer's!) attention on the critical issues (and do not include mention of study variables).

7. Parenthetically refer to tables and figures and avoid statements in which a table or figure is either subject or 
object of a sentence. Parenthetic reference places emphasis on interpretation of the information in the table or figure and not the table or figure.

8. Regularly count words from the Introduction through Discussion.

9. Read the guidelines for publishing in CORR (or any other journal) before submission. Those guidelines generally will need to be met in any case.

\section{Appendix 1}

Content and Organization for Materials and Methods for Studies on Living Patients

These instructions apply when there is a need for Materials and Methods and Results sections. They do not apply to case reports, Orthopaedic-Radiology-Pathology conferences, or selected other unusual articles (eg, survey articles, articles where patient material is used to address nonclinical questions).

Description of Materials and Methods generally should be in past tense. Change passive to active voice when possible but not necessarily every sentence.

No subheadings (in most cases, a subheading merely duplicates the key point of a well-formulated lead sentence).

\section{Paragraph 1}

At the beginning of Materials and Methods, authors must clearly describe the study design in the first paragraph. (Please see our published table of Levels of Evidence for examples of descriptions of clinical study designs.) The study design should complement the questions just raised in the Introduction, and the questions should be inferred from the study design. Ensure the study design describes a method to answer the primary research question. If an IRB statement is appropriate, it can go at the end of the first paragraph, but this is not required since we require it on the title page.

Level I to III must include a description of independent (two or more groups) and dependent (outcome) variables.

Level IV must include a description of dependent (outcome) variables (there is only one group).

Level I to III (one to two paragraphs)

- Study design (sometimes one paragraph) including description of experimental and control subjects

- Power analysis including clinically important effect size
- Total potentially eligible patient base (ie, with the diagnosis or the treatment) including time over which patients enrolled

- Must state whether patients consecutive or selected

- Patient selection methods with inclusion and exclusion criteria

- If a study of a treatment (eg, surgery), list all indications for treatment, any prerequisites, and contraindications

- Randomization scheme if groups randomly assigned

- Numbers of patients excluded and for what reasons (eg, QUORUM algorithm)

- Final numbers of patients studied in each group

- Relevant demographic factors (age, gender, time to operation, etc)

- Comparability of relevant demographic factors if multiple groups

- Minimum and range of followup time

- Number of patients lost to followup

- Ethical (IRB) board approval

Level IV (one paragraph)

- Total potentially eligible patient base (ie, with the diagnosis or the treatment) including time over which patients enrolled

- Must state whether patients consecutive or selected

- Patient selection methods with inclusion and exclusion criteria

- If a study of a treatment (eg, surgery), list all indications for treatment, any prerequisites, and contraindications

- Numbers of patients excluded and for what reasons

- Final numbers of patients

- Relevant demographic factors (age, gender, time to operation, etc)

- If historical controls from the literature for comparison, so state

- Minimum and range of followup time

- Number of patients lost to followup

- Ethical (IRB) board approval

\section{Paragraph 2}

Describe surgical procedures.

\section{Paragraph 3}

Describe outcome measures. Unless measures are widely used (eg, range of motion), you should cite sources that 
validate the measures. If subjective measures dependent upon human observation (eg, clinical examinations, radiographs, histologic slides are used), you must state numbers of observers and parenthetically note initials of observers, whether authors or others (others should be noted in Acknowledgments).

\section{Paragraph 4}

In the final paragraph (or two), explain (and justify if appropriate) all statistical tests. Specify which tests were used to analyze which sets of data. State (and justify if appropriate) level of significance. Note software used (manufacturer, city, state or country).

\section{References}

1. 1999 COPE Report. 2003. Committee on Publication Ethics. Available at: http://www.publicationethics.org.uk/reports/1999. Accessed October 28, 2006

2. 2005 COPE Report. 2005. Committee on Publication Ethics. Available at: http://www.publicationethics.org.uk/reports/2005. Accessed October 28, 2006.

3. American Medical Association Manual of Style: A Guide for Authors and Editors. Chicago, IL: Williams and Wilkins; 1997.

4. Bhandari M, Devereaux PJ, Guyatt GH, Cook DJ, Swiontkowski MF, Sprague S, Schemitsch EH. An observational study of orthopaedic abstracts and subsequent full-text publications. J Bone Joint Surg Am. 2002;84:615-621.

5. Bhandari M, Morrow F, Kulkarni AV, Tornetta P 3rd. Metaanalyses in orthopaedic surgery: a systematic review of their methodologies. J Bone Joint Surg Am. 2001;83:15-24.

6. Brand R. Writing for Clinical Orthopaedics and Related Research. Clin Orthop Relat Res. 2003;413:1-7.

7. Carter SP. Writing for Your Peers. New York, NY: Praeger; 1987.

8. Cowell HR. A brief history of the Journal of Bone and Joint Surgery. Clin Orthop Relat Res. 2000;374:136-144.

9. Cowell HR. Ethical responsibilities of editors, reviewers, and authors. Clin Orthop Relat Res. 2000;378:83-89.

10. Daluiski A, Kuhns CA, Jackson KR, Lieberman JR. Publication rate of abstracts presented at the annual meeting of the Orthopaedic Research Society. J Orthop Res. 1998;16:645-649.

11. Day RA. How to Write, Publish a Scientific Paper. Philadelphia, PA: ISI Press; 1979.

12. DeBakey L. The Scientific Journal: Editorial Policies and Practices: Guidelines for Editors, Reviewers, and Authors. St Louis, MO: CV Mosby; 1976.
13. Derkash RS. History of the Association of Bone and Joint Surgeons. Clin Orthop Relat Res. 1997;337:306-309.

14. Engler RL, Covell JW, Friedman PJ, Kitcher PS, Peters RM. Misrepresentation and responsibility in medical research. $N$ Engl J Med. 1987;317:1383-1389.

15. Huth EJ. How to Write, Publish Papers in the Medical Sciences. Philadelphia, PA: ISI Press; 1982.

16. International Committee of Medical Journal Editors. Uniform requirements for manuscripts submitted to biomedical journals. N Engl J Med. 1997;336:309-315.

17. King LS. Why Not Say It Clearly: A Guide to Expository Writing. Boston, MA: Little, Brown and Co; 1991.

18. Moher D, Schulz KF, Altman D. The CONSORT statement: revised recommendations for improving the quality of reports of parallel-group randomized trials. JAMA. 2001;285:1987-1991.

19. Montori VM, Swiontkowski MF, Cook DJ. Methodologic issues in systematic reviews and meta-analyses. Clin Orthop Relat Res. 2003;413:43-54.

20. Motulsky H. Intuitive Biostatistics. New York, NY: Oxford University Press; 1995.

21. National Institutes of Health. Regulations and Ethical Guidelines: Categories of Research That May Be Reviewed by the Institutional Review Board (IRB) Through an Expedited Review Procedure. 2000. Available at: http://www.nihtraining.com/ ohsrsite/guidelines/45cfr46.html\#46.110. Accessed October 28, 2006.

22. Reidenberg MM. Sponsorship, authorship, and accountability. N Engl J Med. 2002;346:290-292.

23. Relman AS. Lessons from the Darsee affair. $N$ Engl J Med. 1983;308:1415-1417.

24. Relman AS. Responsibilities of authorship: where does the buck stop? N Engl J Med. 1984;310:1048-1049.

25. Relman AS. New "Information for Authors" and readers. $N$ Engl J Med. 1990;323:56.

26. Schechter AN, Wyngaarden JB, Edsall JT, Maddox J, Relman AS, Angell M, Stewart WW. Colloquium on scientific authorship: rights and responsibilities. FASEB J. 1989;3:209-217.

27. Shertzer M. The Elements of Grammar. New York, NY: Macmillan Publishing Co; 1986.

28. Sprague S, Bhandari M, Devereaux PJ, Swiontkowski MF, Tornetta P 3rd, Cook DJ, Dirschl D, Schemitsch EH, Guyatt GH. Barriers to full-text publication following presentation of abstracts at annual orthopaedic meetings. J Bone Joint Surg Am. 2003;85:158-163.

29. Sterne JA, Davey Smith G. Sifting the evidence-what's wrong with significance tests? BMJ. 2001;322:226-231.

30. Stroup DF, Berlin JA, Morton SC, Olkin I, Williamson GD, Rennie D, Moher D, Becker BJ, Sipe TA, Thacker SB. Metaanalysis of observational studies in epidemiology: a proposal for reporting. Meta-analysis Of Observational Studies in Epidemiology (MOOSE) group. JAMA. 2000;283:2008-2012.

31. Strunk W Jr, White EB. The Elements of Style. 3rd ed. New York, NY: Macmillan Publishing Co; 1979.

32. Wright RW, Brand RA, Dunn W, Spindler KP. How to write a systematic review. Clin Orthop Relat Res. 2007;455:23-29. 